\title{
Prognostic Value of Myeloid Marker Positivity and its Association with Prognostic Factors in Pediatric Acute Lymphoblastic Leukemia
}

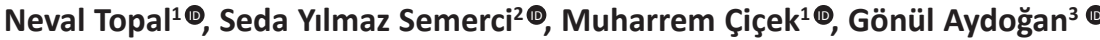

Cite as: Topal N, Yılmaz Semerci S, Çiçek M, Aydoğan G. Prognostic value of myeloid marker positivity and its association with prognostic factors in pediatric acute lymphoblastic leukemia. Trends in Pediatrics 2021;2(3):122-30.

Received: 21 May 2021

Accepted: 19 August 2021

Publication date: 28 September 2021

Keywords: Acute lymphoblastic leukemia, myeloid marker, child

Neval Topal

Health Sciences University Kanuni Sultan Suleyman Training and Research Hospital, Department of Pediatrics, Istanbul - Turkey ORCID: 0000-0003-2771-8744

neomutlu33@gmail.com

S. Yılmaz Semerci 0000-0002-0411-9610

M. Çiçek 0000-0002-8619-2722

G. Aydoğan 0000-0002-3805-531X

\begin{abstract}
Objective: The aim of this study is to investigate the effect of myeloid marker positivity on prognosis and its relationship with classical prognostic factors in acute lymphoblastic leukemia (ALL). Methods: Cases who were newly diagnosed with ALL, followed up in our hospital were included. CD13, CD14 and CD33 were used as myeloid markers by immunophenotyping with flow cytometry in the bone marrow samples. Any parameter higher than $20 \%$ was accepted as positive.Modified BFM-2000 protocol was used in treatment and ALL-BFM-95 residual protocols were used in relapses. Risk groups were determined according to the Trall-BFM2000 protocol. Clinical, laboratory and demographic characteristics of participants such as age, gender, leukocyte count at the time of diagnosis, were all recorded. Patients were divided into three groups according to myeloid markers; those with negative myeloid markers (Group-I), positive one of the myeloid markers (Group-II), those with multiple positive myeloid markers (Group-III). For overall survival, death only was accepted, relapse or death were taken as failure for event free survival (EFS).

Results: A total of 96 ALL cases were included. 44 of the patients were male and 52 were female. Their ages were between 10-204 months and median value was 4.5 years. Nine patients were T-ALL (9.4\%), one had biphenotype ALL (1\%), and 86 had B precursor cell ALL (89.6\%). Group-l had 47 patients. One or more myeloid markers were found to be positive in 49 patients (51.1\%). While 42 of them consisted group-II, group-III had 7 patients. The EFS distribution for all patients was between 43,16-52 months, with a median of 45.58 months. There was no difference between the groups in terms of EFS ( $p$ 0.871).

Conclusion: This study revealed that myeloid markers had no effect on prognosis. When compared with parameters with prognostic significance, no difference was found except between FAB morphology and myeloid markers. Therefore, studies involving more patients are needed to obtain more precise information on the subject.
\end{abstract}

\section{INTRODUCTION}

Hematopoietic malignancies account for more than $40 \%$ of childhood malignancies. While leukemias constitute $25-30 \%$ of childhood cancers, approximately $97 \%$ consists of acute leukemias. Acute lymphoblastic leukemia (ALL) is five times more common in children under the age of 15 than acute myeloblastic leukemia (AML) and the incidence of ALL in 0-14 years of age is 41.4/1.000.000 in our country. ${ }^{1,2}$ While the response and survival rate to ALL treatment was about $10 \%$ in the 1960 s, remission can be achieved at a rate of $80-90 \%$ with current treatment protocols arranged according to the biological characteristics of leukemia cells. ${ }^{1}$

In parallel with the advances in diagnosis and treatment, some clinical and laboratory findings have been found to be effective on prognosis and treatment according to risk has come to the fore. 
Additionally, the age and leukocyte values at the time of diagnosis, biological features such as cytogenesis, immunophenotype and clinical conditions such as central nervous system (CNS) involvement, the presence of an extranodal mass were used in determining the risk groups and the treatments to be applied accordingly. Thus, appropriate chemotherapy is determined for patient groups with low and high recurrence risk. Protocols aimed to apply the treatment with the least side effects as possible and to use more intensive treatment in high-risk groups. ${ }^{3,4}$ However, by the implementation of more effective treatment regimens, some of these prognostic factors have lost their importance and the rapid response to treatment has become the most determining prognostic factor. While the prognosis is shaped according to the treatment, new prognostic information is obtained by evaluating the response to the treatment. ${ }^{4,5}$ Although it is not studied well, existing data are conflicting on the prognostic role of myeloid markers in ALL so far. Therefore, this study aimed to investigate the effect of myeloid marker positivity on prognosis and its relation with classical prognostic factors in childhood ALL.

\section{MATERIALS and METHODS}

Pediatric ALL cases newly diagnosed in a period of four years and treated in our hospital were included in the study. Ethics committee approval was received from study hospital's local ethics committee for this study. Informed consent was obtained from parents and children. Age, gender, leukocyte count at the time of diagnosis, hemoglobin value, hepatomegaly, splenomegaly, lymphadenomegaly (LAM), CNS involvement, mediastinal mass and presence of extramedullary involvement, FAB classification, immunophenotype, associated translocations, risk group, myeloid markers, number of blasts in the smear on treatment day 8 , blast rate in the bone marrow on the 15th and 33rd days, the time of recurrence, if occured, and the time of death if occurred, and the time of treatment initiation were recorded and evaluated by recording clinical and demographic data. Hepatosplenomegaly was noted in case of the abnormal enlargement of liver and spleen according to the age matched precentile values. Death was accepted as only death for overall survival, and relapse or death as failure for eventfree survival (EFS). The follow up period was 52 months. EFS time was taken as the time from diagnosis to relapse or from diagnosis to diseaserelated death.

\section{Diagnosis and Extramedullary Involvement}

Physical examination findings were recorded following detailed anamnesis of all patients with cell morphology and surface antigen compatible with ALL. After routine laboratory analyzes (complete blood count, peripheral blood smear, biochemical tests, virological tests, coagulation tests), bone marrow aspiration was performed, stained with Giemsa and morphologically examined under a light microscope. The percentage of blasts in the bone marrow was determined by FAB criteria (L1: Childhood ALL, L2: Adult type ALL, L3: Burkitt type ALL). Periodic-Acid-Schiff (PAS), Sudan Black and myeloperoxidase staining were performed on bone marrow aspiration (BMA) materials, as well as immunophenotyping with flow cytometry. CD13, CD14 and CD33 as myeloid markers in immunophenotyping; CD19, CD20, CD22, CD24 and CD10 for the B cell line as lymphoid markers; CD3, CD5, CD7 were used for the T cell line. More than $20 \%$ of any parameter and for CD34 a value higher than $10 \%$ was accepted as positive. $t(9 ; 22), t(4 ; 11)$, $t(1 ; 19)$ and $t(12 ; 21)$ were studied for all cases. The presence of a mediastinal mass was investigated by chest radiographs of the patients. CNS involvement was investigated by cytological and biochemical examination of the cerebrospinal fluid (CSF) sample taken by lumbar puncture.

The patients were divided into three groups according to their myeloid markers; Those with negative for myeloid markers (Group I), positive for one of the myeloid markers (Group II), and those with more than one positive myeloid markers (Group III).

\section{Diagnosis and Evaluation of Response to Treatment During Follow-up}

Modified Berlin-Frankfurt-Munster 2000 (BFM-2000) protocol was applied to the patients and ALL BFM-95 Residual protocols were applied in relapse cases. BFM HR blocks were applied to patients who did not respond to treatment or had a partial response and patients included in the high-risk group. The blast 
rate in peripheral blood smear was counted on the 8 th day of the treatment protocol. A blast count of $<1000 / \mathrm{mm}^{3}$ was considered as remission. A leukocyte count of $>100,000 / \mathrm{mm}^{3}$ was defined as hyperleukocytosis. The blast rate in bone marrow preparations on the 15th day was evaluated as M3 bone marrow if it was $25 \%$ or above, $5-24 \%$ as $M 2$ bone marrow, and $<5 \%$ as $\mathrm{M} 1$ bone marrow. A blast rate of $<5 \%$ in bone marrow preparations on the $33 r d$ day showed remission.

\section{Risk Classification}

Our patients were divided into standard, medium and high risk groups according to the Turkish ALLBFM 2000 (TRALL-BFM 2000) protocol.

Standart Risk Group (SRG): Patients meeting all of the following criteria were taken as SRG; age $\geq 1$ year or $<6$ years, initial leukocyte count $<20,000 / \mathrm{mm}^{3}$, leukemic cell count $<1000 / \mathrm{mm}^{3}$ in peripheral blood on the eighth day after 7 days of prednisolone treatment, without T-cell immunology, complete remission on day 33 and cases without $t(9 ; 22)$ or $\mathrm{t}(4 ; 11)$ translocations.

Moderate Risk Group (MRG): Patients with at least one of the criteria were taken as MRG; Cases with leukocytes $\geq 20,000 / \mathrm{mm}^{3}$, age $<1$ year or $\geq 6$ years, or T-cell immunology. Also; should meet all of the following conditions; number of leukemic cells in the peripheral blood on the eighth day is $<1000 / \mathrm{mm}^{3}$, complete remission on the 33 rd day, no t $(9 ; 22)$ and $\mathrm{t}(4 ; 11)$ translocations.

High Risk Group (HRG): Patients with at least one of the criteria were evaluated as HRG; cases with leukemic cell count $>1000 \mathrm{~mm}^{3}$ in peripheral blood on the eighth day, incomplete remission on the 33rd day or positive one of the $t(9 ; 22)$ and $t(4 ; 11)$ translocations.

\section{Statistical Analysis}

Data were presented as mean \pm standard deviation (SD) for parametric tests and median (min-max or 25-75 percentile or interquartile range) for nonparametric tests. Survival analyzes were performed by using the Kaplan-Meier test. Log-rank and Breslow tests were used to compare the survival rates of the groups. SPSS version 21.0 (SPSS, Chicago, IL) was used for statistical analysis. Statistical significance was accepted when the probability (p) value was $<0.05$ and changes were referred to as significant at this $P$ value.

\section{RESULTS}

A total of 96 ALL patients, 44 male and 52 female, diagnosed during a one-year period and aged between 10-204 months (median age 4.5 years) were included in the study (Table 1 ). In the physical examination at the time of first application, hepatosplenomegaly (HSM) was detected in $32 \%$ of the patients and lam in $11.5 \%$. Four patients had a mediastinal mass and were diagnosed with T-cell leukemia. CNS involvement was detected at the time of diagnosis in a patient with biphenotype morphology. Two patients had skeletal involvement and one patient had parotid gland involvement. In laboratory tests, it was seen that $80 \%(n=77)$ of the patients had anemia, $44(46 \%)$ had leukocytosis $\left(>20,000 / \mathrm{mm}^{3}\right)$ and 11 $(11.5 \%)$ of the patients with hyperleukocytosis had a leukocyte count $>100,000 / \mathrm{mm}^{3}$.

Table 1. The distribution of the participants according to the clinical characteristics

Sex
Male
Female
Age (years)
$<1$
$\geq 1-<6$
$\geq 6$
HSM
Yes
No
LAM
Yes
No
Mediastinal mass
Yes
No
SSS involvement
Yes
No
Extramedullary involvement
Yes
No

$44 \quad 45.8$

$52 \quad 54.2$

$2 \quad 2.0$

$64 \quad 66.7$

$30 \quad 31.3$

$31 \quad 32.3$

$65 \quad 67.7$

$11 \quad 11.5$

$85 \quad 88.5$

$4 \quad 4.2$

$1-1.0$

$95 \quad 99.0$

$3 \quad 3.1$

$93 \quad 96.9$

$75 \%$ of BMA smears of the patients were evaluated in FAB-L1 and $25 \%$ in FAB-L2 morphology. In the immunophenotypic evaluation, it was determined that nine patients had T cell phenotype, one patient 
Table 2. Immunephenotypes of the participants

\begin{tabular}{|l|c|c|}
\multicolumn{1}{c|}{} & \multicolumn{2}{c|}{ Number of cases } \\
\hline Immunephenotype & $\mathbf{n}$ & $\%$ \\
\hline B cell & 86 & 89.6 \\
Early Pre B & 6 & 6.3 \\
Pre B & 2 & 2.1 \\
CALLA+ B & 78 & 81.2 \\
T cell & 9 & 9.4 \\
Biphenotype & 1 & 1.0 \\
\hline
\end{tabular}

had biphenotypic characteristics, and 86 (89.6\%) patients had B precursor cell phenotype (Table 2 ).

When the patients were evaluated according to risk groups, $32.3 \%(n=31)$ belonged to the standard risk group, $59.4 \%(n=57)$ to the medium risk and $8.3 \%$ $(n=8)$ to $H R G$.

In cytogenetic examination, three (3.1\%) patients had only $\mathrm{t}(9 ; 22)$, two $(2.1 \%)$ patients had only $\mathrm{t}(4 ; 11)$, any translocations were detected in 85 $(88.7 \%)$ patients and there were no cases where two of them were positive either. The results of six (6.1\%) patients, who died before the final analysis of translocation, could not be reached.

According to myeloid markers, 7 patients had more than one myeloid marker positivity (Group III), 42 patients had one myeloid marker positivity (Group II), and 47 patients had no myeloid marker (Group I). Overall myeloid marker positivity (Group II + Group
III) was $51.1 \%$ ( $n=49)$. Immunophenotype distribution according to the myeloid markers of the patients is summarized in Table 3.

With treatment, 84 (87.5\%) patients developed remission within one month of remission induction therapy. It was observed that a patient who did not proceed to remission was $\mathrm{CD} 13$ positive (group II) at HRG. Although this patient was in remission with BFM HR Block treatment, relapse developed 15 months after the initiation of treatment and the patient who was applied ALL BFM 95 Residive protocol died of sepsis 63 days after relapse, while 11 patients died in the first 30 days due to various reasons. One of these patients was $t(4 ; 11)$ positive, one patient was at HRG, one patient was CD13 positive (Group II) and the other was both $\mathrm{CD} 13$ and CD33 positive (group III). Of the patients who died within the first 30 days, six were at MRG and only one patient was in group II, while the others were found to be group I. The remaining 3 patients were at SRG, and only one patient was in group II, while the others were in group I. One of the two patients followed up in remission developed bone marrow recurrence in the 4th month, and the other had a recurrence of the bone marrow and mediastinal mass in the 12th month. Both of these patients were in MRG and T-cell ALL immunophenotype, one of them was only CD13 positive (Group II), while the other had both $\mathrm{CD} 13$ and $\mathrm{CD} 33$ positive (Group III) (Table 4). These two patients died within two months after relapse. Six patients died within 2-9 months

Table 3. Immunephenotypes of the participants between the groups

Immunephenotype

Early Pre B

Pre $B$

CALLA+B

T cell

Biphenotype

Group I
$\%$

5.2

1.0

39.5

3.2

0.0

Group II
$\%$

1.0

1.0

36.5

5.2

0.0

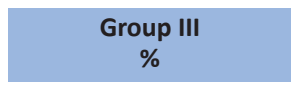

0.0

0.0

5.2

1.0

1.0

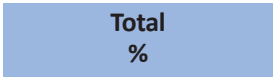

6.3

2.1

81.2

9.4

1.0

Table 4. Current outcomes of the participants between the groups

\begin{tabular}{|l|c|c|}
\hline Current Outcome & $\mathbf{n}$ & $\mathbf{\%}$ \\
\hline Survival & 38 & 39.6 \\
\hline Exitus & 9 & 9.4 \\
\hline Total & 47 & 48.9 \\
\hline
\end{tabular}

\begin{tabular}{|c|c|}
\hline \multicolumn{2}{|c|}{ Group II } \\
\hline $\mathbf{n}$ & $\%$ \\
\hline & \\
\hline 33 & 34.4 \\
\hline 9 & 9.4 \\
42 & 43.8 \\
\hline
\end{tabular}

\begin{tabular}{|c|c|}
\hline \multicolumn{2}{|c|}{ Group III } \\
\hline $\mathbf{n}$ & $\%$ \\
\hline 5 & 5.2 \\
\hline 2 & 2.0 \\
\hline 7 & 7.3 \\
\hline
\end{tabular}

\begin{tabular}{|l|c|}
\hline \multicolumn{3}{|c|}{ Total } \\
\hline $\mathbf{n}$ & $\%$ \\
\hline 76 & 79.2 \\
\hline 20 & 20.8 \\
\hline 96 & 100 \\
\hline
\end{tabular}




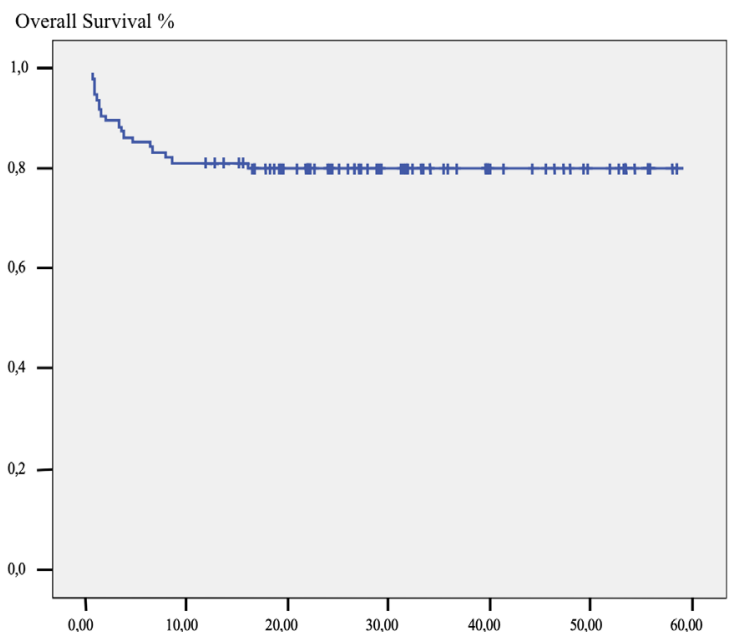

Figure 1. The overall survival rate and the overall survival time were shown as months in Figure 1.

while in remission. $79.2 \%(n=76)$ of our patients survive after 52 months of follow-up. The overall survival rate was $79.9 \%$ and the overall survival time was 47.64 months (43.24-51) (Figure 1). Survival periods of the risk groups were shown in Figure 2 (Figure 2). The 52-month EFS was found to be $80.3 \%$. The EFS distribution for all patients was between 43.16-52 months, with a median of 45.58 months. EFS values according to gender were $76.8 \%$ in boys and $82.4 \%$ in girls ( $p$ 0.061).

Various clinical and laboratory characteristics of our patients (age, gender, hemoglobin value, leukocyte count, HSM, lam, mediastinal mass, CNS involvement, extramedullary involvement, blast morphology, immunophenotype, cytogenesis, blast number in peripheral blood on day 8 , day 15 and 33 , blast rate in bone marrow on the 1st day) was evaluated, it was seen that only immunophenotype and bone marrow response on the 15th day statistically affected the prognosis, while other factors did not

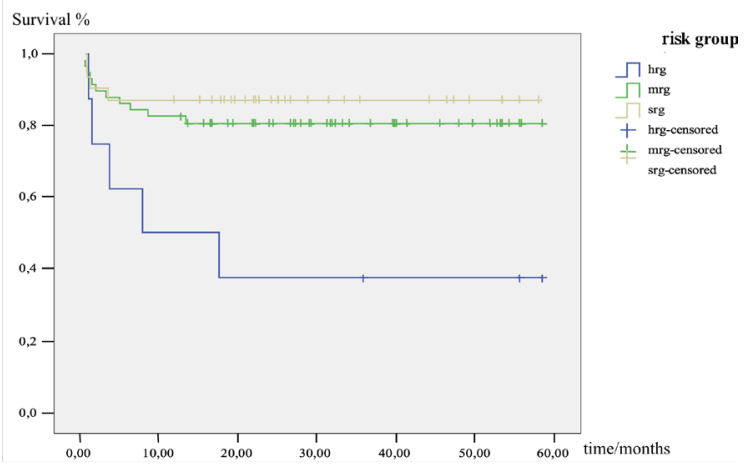

Figure 2. Survival periods of the risk groups were seen in Figure 2.

affect the prognosis. It was observed that the prognosis (EFS 84.9\%) in patients with non-T immunophenotype was better than biphenotype and T-ALL (EFS 29.6\%) ( $p<0.001$ ). Although not used in risk classification, EFS values were found to be significantly different according to the 15th day bone marrow $M 1, M 2$ and $M 3(p<0.001)$. When the EFS of our patients were evaluated according to the risk group, it was seen that the EFS of HRG (37\%) was significantly lower than the other groups ( $p$ 0.008).

None of the cases had all three of the myeloid markers were positive, concurrently. Other myeloid markers were found to be negative in patients with positive CD14. Therefore, in Group III, where two myeloid markers were positive, only CD13 and CD33 were found to be positive either. The effects of these groups on prognosis are given in Table 5. Although the EFS value of Group III seemed to be shorter numerically, no statistically significant difference was found between the EFS. When the groups formed according to myeloid markers were compared with other prognostic factors, it was observed that there was a significant difference between the patients with only FAB-L1 and FAB-L2 blast morphology in

Table 5. Association between the myeloid markers positivity and EFS

\begin{tabular}{|c|c|c|c|c|c|}
\hline & \multicolumn{2}{|c|}{ Number of Cases } & \multirow[b]{2}{*}{ EFS (month) } & \multirow[b]{2}{*}{ EFS (\%) } & \multirow[b]{2}{*}{$p$} \\
\hline & n & $\%$ & & & \\
\hline Group I & 47 & 48.9 & $47.92 \pm 3.1$ & $81.3 \pm 0.05$ & \\
\hline Group II & 42 & 43.8 & $44.44 \pm 3.2$ & $77.7 \pm 0.06$ & 0.871 \\
\hline Group III & 7 & 7.3 & $25.87 \pm 4.89$ & $68 \pm 0.1$ & \\
\hline
\end{tabular}


terms of distribution of the groups ( $p$ 0.012). It was observed that the positive myeloid markers of our patients did not affect the peripheral blood smear and bone marrow blast response. It was found that mortality rates in Group I, II and III did not differ. The number of patients with relapse was very low, and there was no significant difference between the groups in terms of relaps rates.

\section{DISCUSSION}

Acute leukemias are the most common malignant diseases in childhood. Many factors such as age, gender, leukocytosis and cytogenetic values have prognostic importance in childhood ALL. These prognostic factors are also used in classifying patients into risk groups. Given relapses can occur in even patients without poor prognostic factors, current prognostic evaluation is required to be improved. Therefore, it is necessary to search for new prognostic factors in childhood ALL. ${ }^{6}$

In series including larger patient population, the incidence of ALL in male patients was higher than girls, while it was found to be $45.8 \%$ in this study. ${ }^{7}$ Boys are known to have a worse prognosis than girls who receive the same treatment. ${ }^{8}$ Similarly, in this study, although the EFS of males was lower than females, a statistically significant difference was not found, probably due to our relatively low number of cases.

Age at the time of diagnosis is a notable prognostic parameter in ALL. ${ }^{9}$ The worst prognosis is seen under one year of age, related to the certain features such as CNS involvement, high blast load and slower response to treatment are common in this age group. ${ }^{9}$ Especially, under 3 months has the worst prognosis, while the prognosis is better in the range of 6-12 months..$^{1,4}$ The best prognosis is seen between the ages of 2-6 in all age groups. ${ }^{9}$ In our study, the median age at the time of diagnosis was 4.5 years and had similar results with studies in Germany and Korea. ${ }^{10,11}$ Besides, the median age was stated to be around 6 years in different studies. ${ }^{12-14}$

Leukocyte count at the time of diagnosis is also defined as another prognostic factor, of which higher rate is associated with an increased risk of recurrence, especially in B-ALL. ${ }^{9}$ Significant higher or lower leukocyte counts at the time of diagnosis may delay the diagnosis of ALL. Leukocytosis $\left(>20,000 / \mathrm{mm}^{3}\right)$ was not detected in more than half of our cases. There are different approaches to the cut-off value of leukocyte count. ${ }^{12,13}$ When taken the commonly used value of $20,000 / \mathrm{mm}^{3}$ as cut-off in our study, it was seen that there was no difference between the EFS of the cases with the leukocyte count above $20.000 / \mathrm{mm}^{3}$ and the cases with less than $20,000 /$ $\mathrm{mm}^{3}$.

While anemia, which is present in more than $80 \%$ of the patients at the time of diagnosis, indicates that leukemia has been present for a relatively long time, normal hemoglobin $(\mathrm{Hb})$ values suggest a rapid course of leukemia. It is thought that patients presenting with higher $\mathrm{Hb}$ levels or mild anemia are detected at an early stage of the disease. Therefore, they may be more susceptible to chemotherapeutic interventions. ${ }^{1,2,15,16}$ In a study investigating the effect of $\mathrm{Hb}$ level on prognosis, complete remission rates were reported as $63 \%$ when $\mathrm{Hb}$ level was $<8 \mathrm{~g} / \mathrm{dl}, 70 \%$ between $10-12 \mathrm{~g} / \mathrm{dl}$ and $60 \%$ when $>12 \mathrm{~g} / \mathrm{dl}$. It has been stated that the level does not affect the life span. ${ }^{17}$ In our study, there was no statistically significant effect of $\mathrm{Hb}$ level on EFS.

In the literature, fever, organomegaly, lymphadenopathy and pallor have been defined as the most common clinical features. ${ }^{16}$ Patients with hepatomegaly and/or splenomegaly at the time of ALL diagnosis, had been shown to have an almost four times higher risk of recurrence. ${ }^{18}$ Studies examining the relationship between splenomegaly/ hepatomegaly and survival have conflicting results. ${ }^{19}$ In our study, HSM, LAM, and the presence of a mediastinal mass, which are among the poor prognostic parameters, was not found to be effective on prognosis due to the possible low number of patients.

In the past, all lymphoblasts were classified according to $F A B$ criteria. Currently, this classification is not recommended due to the lack of independent prognostic significance and being subjective. However, it still has a place in clinical practice, as it can provide diagnostic accuracy in some cases. Additionally, the FAB system is preferred in developing 
countries such as our country, given its advantages of being practical and not requiring much resources. ${ }^{16,20}$ L1 morphology is more common in children (about $74 \%$ ) than adults (about $66 \%$ ). ${ }^{21}$ In accordance with the literature, distribution of our patients in L1 and L2 according to the morphological classification was $75 \%$ and $25 \%$, respectively. When the sole effect of blast morphology on prognosis was examined, it was seen that the EFS values of $L 1$ and L2 did not differ.

Approximately $2-3 \%$ of children with ALL, have t(4; 11) and $t(9 ; 22)$, which is known as Philadelphia chromosome, was found in $2.9 \%$ of them. ${ }^{19}$ These translocations are known to be associated with poor prognosis. ${ }^{19,20}$ In this study, t $(9 ; 22)$ and t $(4 ; 11)$ was found to be $3.1 \%$ and $2 \%$, respectively. However, due to the low number of patients with positive translocations in our study, a statistical evaluation could not be made.

Immunophenotyping in leukemic lymphoblast cells forms the basis of diagnostic evaluation, since these cells lack specific morphological and cytochemical properties. Expression of $C D$ markers is widely used to classify hematological malignancies, including leukemia and lymphoma.,222 This study evaluated myeloid surface antigens such as CD13, CD14 and CD33, which are not found in normal $T$ or $B$ lymphocytes, with a lower limit of $20 \%$ in fluorescence analysis. In our series, the myeloid marker positivity with $51.1 \%$ is much higher than the $4-22 \%$ reported in the literature. Uckun et al. reported that they used only CD13 and CD33 antigens with a lower limit of $30 \%$ in fluorescence analysis in 1557 patients and their myeloid antigen positivity was $16.6 \%{ }^{23}$ Using the lower limit of $20 \%$, similar to our study, Putti et al. found the myeloid marker positivity to be $32 \%$ in their series of 908 patients. ${ }^{24}$. The researchers stated that screening CD13 and CD33 myeloid markers will reveal the presence of all myeloid antigens in childhood ALL. ${ }^{24}$ We tried to create a wider panel by looking at CD14 in addition to these antigens. In this study, CD13 was found to be the most detected myeloid marker in $44 \%$ of patients. CD14 and CD33 were found positive in $4 \%$ and $9 \%$, respectively. When our patients with T-biphenotype and B precursor cell ALL were evaluated according to the distribution of myeloid marker positivity, it was observed that there was no statistically significant difference ( $p$ 0.52). Similar to our results, Putti et al. found that the percentage of myeloid marker positivity was similar between these two immunophenotypes. ${ }^{24}$ When the association of ALL and myeloid marker positivity was compared with other prognostic values such as age, gender, high leukocyte count, hemoglobin value, HSM, presence of translocations and immunophenotype, no statistically significant difference was found. When evaluated only in terms of blast morphology, it was observed that myeloid marker positivity was higher in L2 morphology, which was considered to show a relatively poor prognosis ( $p$ 0.012). Uckun et al. showed that patients with myeloid antigen negative B precursor cell ALL have more clinical and laboratory values such as higher leukocyte count, splenomegaly and low platelet count, which show worse prognosis compared to patients with myeloid antigen positive B precursor cell ALL. ${ }^{23}$ There are also previous studies, from different countries, which obtained data similar to ours. ${ }^{24,25}$ On the other hand, Fink et al. reported that myeloid antigen positive patients presented with higher leukocyte count; but EFS of these cases did not differ than others. ${ }^{26}$

Clinical importance of myeloid marker positivity has been presented in the literature since 1990. Although most of the studies showed that myeloid markers have no effect on the prognosis, the results are contradicting. Wiersma et al. stated that myeloid antigen positivity was the most important indicator of poor prognosis, while other studies reported that myeloid antigen positive patients had better prognosis than myeloid antigen negative ones. ${ }^{26,27}$ Mejstríková et al. showed that CD13 and CD33 markers have prognostic value and 5-year EFS is lower in myeloid antigen negative ones. ${ }^{28}$ Despite it was not evaluated a prognostic factor, children with myeloid markers and Philedelpia choromosome positive ALL was shown to have a poor early response to treatment, which resulted in a low CR rate. ${ }^{29}$

However, in their study with a large ALL population, Uckun et al. found that 4-year EFS was similar in both myeloid antigen positive and negative ones. ${ }^{23}$ Pui et al. reported that the myeloid antigen found in 105 of 334 patients with newly diagnosed ALL, but this did not affect EFS although it was associated with 
specific genetic anomalies. ${ }^{29}$ There are also several studies reporting that myeloid antigen positivity does not affect the prognosis. ${ }^{22,24,25,31,32}$ In our study, it was seen that EFS was not affected by myeloid markers.

\section{Limitations}

This study has some limitations such as restricted number of participants. Including larger numbers of participants may provide a more strengthened study.

\section{CONCLUSION}

This study revealed that myeloid markers had no effect on ALL prognosis. When compared with other prognostic parameters, no difference was found except $F A B$ morphology. In conclusion, myeloid antigen positivity is a common condition in patients with childhood ALL, and its prognostic significance is controversial. Although there is no objective data to explain the positive myeloid markers in the patient group with FAB-L2 blast morphology showing only a relatively poor prognosis, it was thought that the low number of our patients led to this situation. Therefore, studies involving more patients are needed to obtain more precise information on the subject.

Ethics Committee Approval: The study was approved by local Ethics Committee of study hospital (Ethics Committee approval number: 154/2008.06.26).

Conflict of Interest: The authors have no conflicts of interest to disclose.

Funding: No funding was secured for this study.

Informed Consent: Informed consent was obtained from the parents prior to participation in the study.

\section{REFERENCES}

1. Yöntem A, Bayram İ. Çocukluk çağında akut lenfoblastik lösemi. Arşiv Kaynak Tarama Dergisi. 2018;27:483-99. https://doi.org/10.17827/aktd.397762

2. Uzunhan TA, Karakaş Z. Çocukluk çağı akut lenfoblastik Iösemisi. Çocuk Dergisi. 2012;12:6-15.

3. Smith M, Arthur D, Camitta B, et al. Uniform approach to risk classification and treatment assignment for children with acute lymphoblastic leukemia. J Clin Oncol. 1996;14:18-24. https://doi.org/10.1200/JCO.1996.14.1.18

4. Rabin KR, Gramatges MM, Margolin JF, Poplack DG. Acute Lymphoblastic Leukemia. In: Pizzo PA, Poplack
DG, editors. Principles and practice of pediatric oncology. 7th ed. Philadelphia: Lippincott Williams \& Wilkins; 2015. p. 463-96.

5. Gaynon PS, Desai AA, Bostrom BC, et al. Early response to therapy and outcome in childhood acute lymphoblastic leukemia: a review. Cancer. 1997;80:1717-26.

https://doi.org/10.1002/(SICI)1097-0142(19971101) 80:9<1717::AID-CNCR4>3.0.CO;2-B

6. Donadieu J, Hill C. Early response to chemotherapy as a prognostic factor in childhood acute lymphoblastic leukaemia: a methodological review. British Journal of Haematology. 2001;115:34-45. https://doi.org/10.1046/j.1365-2141.2001.03064.x

7. Siegel DA, Henley SJ, Li J, Pollack LA, Van Dyne EA, White A. Rates and Trends of Pediatric Acute Lymphoblastic Leukemia - United States, 2001-2014. MMWR Morb Mortal Wkly Rep. 2017;66:950-4. https://doi.org/10.15585/mmwr.mm6636a3

8. Ishii E, Eguchi $H$, Matsuzaki A, Koga H, Yanai F, Kuroda $\mathrm{H}$, et al. Outcome of acute lymphoblastic leukemia in children with AL90 regimen: impact of response to treatment and sex difference on prognostic factors. Med Pediatr Oncol. 2001;37:10-9. https://doi.org/10.1002/mpo.1156

9. Vrooman LM, Silverman LB. Treatment of childhood acute lymphoblastic leukemia: Prognostic factors and clinical advances. Curr Hematol Malig Rep. 2016;11: 385-94. https://doi.org/10.1007/s11899-016-0337-y

10. Erdmann F, Kaatsch P, Zeeb H, Roman E, Lightfoot T, Schüz J. Survival from childhood acute lymphoblastic leukaemia in West Germany: does socio-demographic background matter? Eur J Cancer. 2014;50:1345-53. https://doi.org/10.1016/j.ejca.2014.01.028

11. Kim SJ, Park MH, Lee JW, et al. Neurocognitive outcome in survivors of childhood acute lymphoblastic leukemia: experience at a tertiary care hospital in Korea. J Korean Med Sci. 2015;30:463-9. https://doi.org/10.3346/jkms.2015.30.4.463

12. Jaime-Pérez JC, García-Arellano G, Herrera-Garza JL, Marfil-Rivera LJ, Gómez-Almaguer D. Revisiting the complete blood count and clinical findings at diagnosis of childhood acute lymphoblastic leukemia: 10-year experience at a single center. Hematol Transfus Cell Ther. 2019;41:57-61. https://doi.org/10.1016/j.htct.2018.05.010

13. Lustosa de Sousa DW, de Almeida Ferreira FV, Cavalcante Félix FH, de Oliveira Lopes MV. Acute lymphoblastic leukemia in children and adolescents: prognostic factors and analysis of survival. Rev Bras Hematol Hemoter. 2015;37:223-9. https://doi.org/10.1016/j.bjhh.2015.03.009

14. Fadoo Z, Nisar I, Yousuf F, et al. Clinical features and induction outcome of childhood acute lymphoblastic leukemia in a lower/middle income population: A multi-institutional report from Pakistan. Pediatr Blood Cancer. 2015;62:1700-8. https://doi.org/10.1002/pbc.25583

15. Teuffel O, Stanulla M, Cario G, et al. Anemia and survival in childhood acute lymphoblastic leukemia. Haematologica. 2008;93:1652-7. https://doi.org/10.3324/haematol.13156

16. Kakaje A, Alhalabi MM, Ghareeb A, et al. Rates and 
trends of childhood acute lymphoblastic leukaemia: an epidemiology study. Sci Rep. 2020;10:6756.

https://doi.org/10.1038/s41598-020-63528-0

17. Harousseau JL, Tobelem G, Schaison G, et al. High risk acute lymphocytic leukemia: a study of 141 cases with initial white blood cell counts over 100,000/cu mm. Cancer. 1980;46:1996-2003. https://doi.org/10.1002/1097-0142(19801101) 46:9<1996::AID-CNCR2820460917>3.0.CO;2-R

18. Jaime-Pérez JC, Pinzón-Uresti MA, Jiménez-Castillo RA, Colunga-Pedraza JE, González-Llano Ó, GómezAlmaguer D. Nükse of childhood acute lymphoblastic leukemia and outcomes at a reference center in Latin America: organomegaly at diagnosis is a significant clinical predictor. Hematology. 2018;23:1-9. https://doi.org/10.1080/10245332.2017.1333294

19. Bahoush G, Nojoomi M. Frequency of cytogenetic findings and its effect on the outcome of pediatric acute lymphoblastic leukemia. Med Arch. 2019;73:311-5. https://doi.org/10.5455/medarh.2019.73.311-315

20. PDQ Pediatric Treatment Editorial Board. Childhood Acute Lymphoblastic Leukemia Treatment $\left(\mathrm{PDQ}^{\circledR}\right)$ : Health Professional Version. PDQ Cancer Information Summaries. Bethesda (MD): National Cancer Institute (US); 2021.

21. Barazanchi A, Ali ZA, Al Sami A, Naema NF. Haematological and cytomorphological study of acute lymphoblastic leukemia [ALL]. Bahrain Medical Bulletin. 2005;27:175-9.

22. Kavianpour M, Ketabchi N, Saki N. Prognostic significance of aberrant expression of $C D$ markers in acute lymphoblastic leukemia. memo-Magazine of European Medical Oncology. 2017;10:164-9. https://doi.org/10.1007/s12254-017-0324-6

23. Uckun FM, Sather HN, Gaynon PS, et al. Clinical features and treatment outcome of children with myeloid antigen positive acute lymphoblastic leukemia: a report from the Children's Cancer Group. Blood. 1997;90:28-35.

24. Putti MC, Rondelli R, Cocito MG, et al. Expression of myeloid markers lacks prognostic impact in children treated for acute lymphoblastic leukemia: Italian experience in AIEOP-ALL 88-91 studies. Blood. 1998;92:795-801.

https://doi.org/10.1182/blood.V92.3.795

25. Ng SM, Ariffin WA, Lin HP, Chan LL, Chin YM. Clinical features and treatment outcome of children with myeloid antigen coexpression in B-lineage acute lymphoblastic leukemia: a study of 151 Malaysian children. J Trop Pediatr. 2000;46(2):73-8. https://doi.org/10.1093/tropej/46.2.73

26. Fink FM, Köller $\mathrm{U}$, Mayer $\mathrm{H}$, et al. Prognostic significance of myeloid-associated antigen expression on blast cells in children with acute lymphoblastic leukemia. The Austrian Pediatric Oncology Group. Med Pediatr Oncol. 1993;21(5):340-6. https://doi.org/10.1002/mpo.2950210506

27. Wiersma SR, Ortega J, Sobel E, Weinberg KI. Clinical importance of myeloid-antigen expression in acute lymphoblastic leukemia of childhood. N Engl J Med. 1991;324(12):800-8.

https://doi.org/10.1056/NEJM199103213241204

28. Mejstríková E, Kalina T, Trka J, Starý J, Hrusák O. Correlation of CD33 with poorer prognosis in childhood ALL implicates a potential of anti-CD33 frontline therapy. Leukemia. 2005;19(6):1092-4. https://doi.org/10.1038/sj.leu.2403737

29. Xue YJ, Wu J, Zuo YX, Jia YP, Lu AD, Zhang LP. Zhonghua Xue Ye Xue Za Zhi. Prognostic factors of pediatric patients with $\mathrm{Ph}$-positive acute lymphoblastic leukemia. Chinese Journal of Hematology. 2018;39(3):219-24.

30. Pui $\mathrm{CH}$, Rubnitz JE, Hancock ML, et al. Reappraisal of the clinical and biologic significance of myeloidassociated antigen expression in childhood acute lymphoblasticleukemia.J Clin Oncol. 1998;16(12):376873. https://doi.org/10.1200/JCO.1998.16.12.3768

31. Drexler HG, Thiel E, Ludwig WD. Review of the incidence and clinical relevance of myeloid antigen-positive acute lymphoblastic leukemia. Leukemia. 1991;5(8): 637-45.

32. Lee JW, Cho B. Prognostic factors and treatment of pediatric acute lymphoblastic leukemia. Korean J Pediatr. 2017;60:129-37.

https://doi.org/10.3345/kjp.2017.60.5.129 